\section{THE DIAGNOSTIC ACCURACY OF BIOCHEMICAL MEASUREMENTS IN SCREENING FOR METABOLIC BONE DISEASE IN PRETERM INFANTS: A SYSTEMATIC REVIEW}

\author{
F. Visser, A.J. Sprij, F. Brus \\ Paediatrics, Juliana Children's Hospital, Haga \\ Hospital, The Hague, The Netherlands
}

Background and aims: The use of biochemical tests in serum and urine for detecting metabolic bone disease (MBD) in preterm infants remains questionable. The aim is to determine the reliability of biochemical measurements in serum and urine for diagnosing MBD in preterm infants by means of a systematic review of the current literature.

Methods: Relevant articles were identified by searching in Medline, the Cochrane library and Embase and by screening reference lists. Studies regarding $\mathrm{MBD}$ in preterm infants that compare serum and/or urine measurements to imaging techniques were included. Screening for inclusion, data extraction and quality assessment were carried out by two authors independently. No meta-analysis was performed, because of extensive clinical heterogeneity and methodological limitations in the majority of the studies. All included studies underwent qualitative data analysis and were given an overall score of "poor", "moderate" or "high" quality.

Results: Twenty-five studies were included. The methodological quality is poor in 14, moderate in 11 and high in none of the studies. Studies show conflicting results regarding serum alkaline phosphatase (AP) and serum phosphate as indicators of MBD. Serum calcium is not correlated with MBD. Urinary calcium and phosphate concentrations might be useful as markers of MBD in stable clinical circumstances but few studies have been performed.

Conclusions: Our systematic review shows insufficient evidence to use serumAP and phosphate concentrations as reliable indicators of MBD in preterm infants. Serum calcium is not a useful indicator. Urinary calcium and phosphate excretion might be useful but require more research.

\section{WATERBABIES: A 10 YEAR REVIEW}

\author{
K. Tanney ${ }^{1}$, R. Jess ${ }^{2}$, M. Hogan ${ }^{1}$ \\ ${ }^{1}$ Neonatal Unit, ${ }^{2}$ Midwife-led Unit, Craigavon Area \\ Hospital, Portadown, UK
}

Background: Waterbirth has become a popular mode of delivery for women with low-risk pregnancies. Despite historic concern about neonatal thermoregulation, infection and respiratory distress, recent Cochrane review reported no difference between land and waterbirths in 5-minute Apgar scores, neonatal unit admissions or infection rates. Waterbirth has been offered to selected mums in the midwifery-led unit of a Northern Ireland district general hospital since August 2000. This retrospective study assessed the comparative admission rates of "waterbabies" over this 10-year period.

Methods: Using data from "low-risk" maternity records, neonatal unit admission diaries and baby charts, details of babies admitted to the neonatal unit since August 2000 were reviewed. Comparisons were made between land and waterbirth admission rates, diagnoses and Apgar scores.

Results: 6109 deliveries have taken place in this midwifery-led unit since August 2000, $27 \%$ of which are waterbirths. $2.2 \%$ of babies born in water and $2.5 \%$ on land have required NICU admission. Respiratory distress (RDS, TTN or pneumonia) comprised $40 \%$ of waterbirth and $31 \%$ of landbirth admissions, and polycythaemia $25 \%$ vs $17 \%$. $5 \%$ of landbirth admissions were due to neonatal encephalopathy. The mean 5-minute Apgar scores and sepsis rates were equal for both land and waterbirths.

Conclusions: This midwifery-led unit shows no increased admission rate for babies born by waterbirth. Amongst the waterbirths there are greater percentages of admissions due to respiratory distress and polycythaemia, but none with neonatal encephalopathy. This study echoes the findings of recent Cochrane review; waterbirth offers a safe mode of delivery for women with lowrisk pregnancies. 